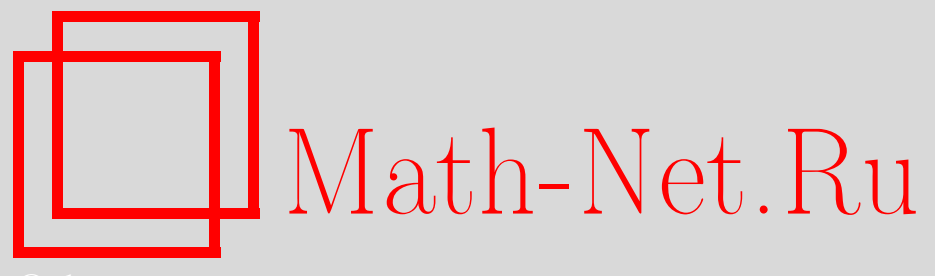

А. С. Кузьмин, В. Т. Марков, А. А. Нечаев, А. Б. Шишков, Приближение булевых функций мономиальными, Дискрет. матем., 2006, том 18, выпуск 1, 9-29

DOI: https://doi.org/10.4213/dm29

Использование Общероссийского математического портала Math-Net.Ru подразумевает, что вы прочитали и согласны с пользовательским соглашением http://www. mathnet.ru/rus/agreement

Параметры загрузки:

IP : 18.209 .158 .208

26 апреля 2023 г., 14:29:55 


\title{
Приближение булевых функций мономиальными
}

\author{
() 2006 г. А. С. Кузьмин, В. Т. Марков,
}

А. А. Нечаев, А. Б. Шишков

Каждая булева функция от $n$ переменных отождествляется с функцией $F: Q \rightarrow P$, где $Q=G F\left(2^{n}\right), P=G F(2)$. Ранее Йоссером и Гонгом для $n=2 \lambda$ было установлено существование функций $F$, которые одинаково плохо приближаются не только линейными функциями (функциями $\operatorname{tr}(\mu x)$, где $\mu \in Q^{*}$ и $\operatorname{tr}: Q \rightarrow P$ есть функция след), но и собственными мономиальными функциями (функциями $\operatorname{tr}\left(\mu x^{\delta}\right)$, где $\left.\left(\delta, 2^{n}-1\right)=1\right)$ ). Такие функции $F$ названы гипер-бент-функциями (ГБ-функциями, ГБФ), и описан класс ГБФ со свойством $F(0)=0$, непустой при любом $n=2 \lambda$. Это функции вида $F(x)=G\left(x^{2^{\lambda}-1}\right)$ такие, что уравнение $F(x)=1$ имеет ровно $\left(2^{\lambda}-1\right) 2^{\lambda-1}$ решений в $Q$. В данной работе указаны существенные ограничения на параметры произвольной ГБФ, показывающие, что класс ГБ-функций существенно меньше класса бент-функций. В частности, показано, что любая ГБ-функция есть бент-функция степени нелинейности $\lambda$ и при некоторых $n$ (например, в случае, когда $\lambda>2,2^{\lambda}-1$ - простое число или $\left.\lambda \in\{4,9,25,27\}\right)$ класс ГБ-функций исчерпывается функциями вида $F(x)=G\left(x^{2^{\lambda}-1}\right)$, описанными Йоссером и Гонгом. Для $n=4$, кроме 10 ГБ-функций указанного вида, существует еще 18 других ГБ-функций со свойством $F(0)=0$. Вопрос о существовании других гипер-бент-функций при $n>4$ остается открытым.

Работа выполнена при поддержке Российского фонда фундаментальных исследований, проекты 05-01-01048 и 05-01-01018, и программой Президента Российской Федерации поддержки ведущих научных школ, гранты НШ 1910.2003.1 и НШ 2358.2003.9.

\section{1. Введение}

В различных теоретических и прикладных задачах возникает необходимость найти приближение некоторой функции на конечном множестве функцией из заданного класса функций. Под приближением понимается функция из этого класса, значения которой совпадают со значениями исходной функции на максимальном числе наборов аргументов. Наиболее глубоко изучены с этой точки зрения булевы функции и их линейные приближения $[1,3,4]$.

Всюду далее используются следующие обозначения: $P=G F(2)-$ поле из двух элементов, $Q=G F\left(2^{n}\right)$ - расширение поля $P$ степени $n$. Булевы функции от $n$ переменных рассматриваются как функции $F: Q \rightarrow P$ (детали см. в следующем разделе). В качестве основного параметра, характеризующего степень близости функции $F$ и приближающей 
ее функции (статистического аналога) $A: Q \rightarrow P$ используется наиболее удобная для изучения арифметическая сумма

$$
\Delta(F, A)=\sum_{x \in Q}(-1)^{F(x)+A(x)},
$$

где $(-1)^{0}=1,(-1)^{1}=-1$. Через этот параметр хорошо выражаются расстояние Хемминга между функциями $F$ и $A$ :

$$
d(F, A)=\frac{2^{n}-\Delta(F, A)}{2}
$$

и вероятность совпадения значений функций при случайном равновероятном выборе аргумента:

$$
\mathbf{P}(F=A)=\frac{2^{n}-d(F, A)}{2^{n}}=\frac{1}{2}+\frac{\Delta(F, A)}{2^{n+1}} .
$$

Условие $\Delta(F, A)<0$ означает, что вместо функции $A$ нужно выбрать в качестве приближения $F$ функцию $A+1$, так как $\Delta(F, A+1)=-\Delta(F, A)$.

В качестве показателя эффективности приближения функции $F$ функциями из некоторого класса функщий $\mathscr{A}$ выбирается величина

$$
\Delta(F, \mathscr{A})=\max \{|\Delta(F, A)|: A \in \mathscr{A}\} .
$$

При этом величина

$$
\frac{1}{2}+\frac{\Delta(F, \mathscr{A})}{2^{n+1}}
$$

есть вероятность совпадения значений функции $F$ и ее наилучшего приближения в классе $\mathscr{A} \cup \mathscr{A}+1$ при случайном равновероятном выборе значений аргумента.

Параметр

$$
\Delta \mathscr{A}=\min \{\Delta(F, \mathscr{A}): F: Q \rightarrow P\}
$$

характеризует возможность класса $\mathscr{A}$ аппроксимировать произвольную булеву функцию, через него выражается радиус покрытия rs $(\mathscr{A})$ пространства Хемминга $P \mathcal{Q}$ всех булевых функций от $n$ переменных системой функций $\mathscr{A} \cup \mathscr{A}+1$ :

$$
\operatorname{rs}(\mathscr{A})=\frac{2^{n}-\Delta \mathscr{A}}{2}
$$

Наиболее полно с этой точки зрения исследован класс линейных функщий $\mathscr{L}=\mathscr{L}(Q \rightarrow P)$, то есть функций $L_{a}: Q \rightarrow P$ вида

$$
L_{a}(x)=\operatorname{tr}(a x), \quad a \in Q .
$$

Здесь

$$
\operatorname{tr}(x)=\operatorname{tr}_{P}^{Q}(x)=\sum_{l=0}^{n-1} x^{2^{l}}
$$

- след из поля $Q$ в поле $P$. Известно (см., например, [3]), что $\Delta \mathscr{L} \geqslant 2^{n / 2}$. Очевидно, что если последнее неравенство является равенством, то $n=2 \lambda-$ четное число. В последнем случае, действительно,

$$
\Delta \mathscr{L}=2^{\lambda},
$$


то есть для любой булевой функции от $n$ переменных существует линейный статистический аналог, для которого вероятность совпадения значений с исходной функцией не меньше величины $1 / 2+(1 / 2)^{\lambda+1}$. Класс функций $F$, для которых эта оценка достигается, то есть функщий, удовлетворяющих равенству

$$
\Delta(F, \mathscr{L})=2^{\lambda}
$$

исследовался различными авторами. В настоящее время для таких функций используется термин бент-функщии $[3,4]$. Из указанных работ известны, в частности, следующие свойства бент-функций.

Предложение 1. Для булевой функиии $f$ om $n=2 \lambda$ переменных, следуючие утверждения равносильны:

(a) $f$-бент-функция;

(б) $\left|\Delta\left(F, L_{a}\right)\right| \leqslant 2^{\lambda}$ для любой линейной функции $L_{a}$;

(в) $\left|\Delta\left(F, L_{a}\right)\right|=2^{\lambda}$ для любой линейной функции $L_{a}$.

Если $f$ - бент-функция, то ее степень нелинейности удовлетворяет неравенству

$$
\operatorname{deg} f \leqslant \lambda \text {. }
$$

В [12] изучалась возможность приближения булевых функщий в классе функций $L_{a}^{(\delta)}: Q \rightarrow P$ вида

$$
L_{a}^{(\delta)}(x)=\operatorname{tr}\left(a x^{\delta}\right), \quad a \in Q, \quad \delta \in\left\{1, \ldots, 2^{n}-1\right\}, \quad\left(\delta, 2^{n}-1\right)=1,
$$

которые мы называем собственными мономиальными функциями. Этот класс включает в себя составной частью класс линейных функций. Было показано, что существуют булевы функции $F$, названные гипер-бент-функциями, которые одинаково плохо приближаются всеми собственными мономиальными функциями, и построен довольно большой класс гипер-бент-функций для каждого $n=2 \lambda$ (см. здесь разд. 4).

В данной работе (разд. 3) показано, что гипер-бент-функции удовлетворяют некоторым дополнительным условиям, существенно ограничивающим их совокупность, например, все они - бент-функции степени нелинейности $\lambda$. Отсюда следует, что для ряда бентфункций в классе мономиальных функций существуют статистические аналоги, имеющие вероятность совпадения со значениями исходной функции большую, чем $1 / 2+(1 / 2)^{\lambda+1}$. Кроме того, показано (разд. 4), что в некоторых случаях (например, если $2^{\lambda}-1$ - простое число) не существует гипер-бент-функций, отличных от тех, которые описаны в [12].

\section{2. Представление булевых функций от $n$ переменных многочленами над полем $Q=G F\left(2^{n}\right)$}

Пусть $\varepsilon_{0}, \varepsilon_{1}, \ldots, \varepsilon_{n-1}-$ базис поля $Q=G F\left(2^{n}\right)$, рассматриваемого как пространство над полем $P=G F(2)$, и $\omega_{0}, \omega_{1}, \ldots, \omega_{n-1}-$ базис $Q_{P}$, двойственный к базису $\varepsilon_{0}, \varepsilon_{1}, \ldots, \varepsilon_{n-1}$ (см. [8]), то есть базис, удовлетворяющий условию

$$
\operatorname{tr}\left(\varepsilon_{j} \omega_{l}\right)= \begin{cases}1, & \text { если } j=l \\ 0, & \text { если } j \neq l\end{cases}
$$


Тогда для любого набора булевых величин $x_{0}, x_{1}, \ldots, x_{n-1}$ однозначно определен элемент $x$ поля $G F\left(2^{n}\right)$

$$
x=\sum_{l=0}^{n-1} x_{l} \varepsilon_{l} .
$$

При этом в силу двойственности базисов справедливы соотношения

$$
x_{l}=\operatorname{tr}\left(\omega_{l} x\right), \quad l \in\{0,1, \ldots, n-1\} .
$$

При справедливости (2.1) для произвольной булевой функщии $f\left(x_{0}, x_{1}, \ldots, x_{n-1}\right)$ получаем равенство

$$
f\left(x_{0}, x_{1}, \ldots, x_{n-1}\right)=f\left(\operatorname{tr}\left(\omega_{0} x\right), \operatorname{tr}\left(\omega_{1} x\right), \ldots, \operatorname{tr}\left(\omega_{n-1} x\right)\right),
$$

ставящее ей в соответствие функщию $F: Q \rightarrow P$, определяемую равенством

$$
F(x)=f\left(\operatorname{tr}\left(\omega_{0} x\right), \operatorname{tr}\left(\omega_{1} x\right), \ldots, \operatorname{tr}\left(\omega_{n-1} x\right)\right) .
$$

Любая такая функция однозначно представляется многочленом над $Q$ степени не выше $2^{n}-1$, причем, так как все значения функщии $F(x)$ на $Q$ принадлежат подполю $P$, это представление может быть записано в виде

$$
F(x)=\operatorname{tr}(\Phi(x))
$$

где $\Phi(x)$ - некоторый полином над полем $Q$, который, вообще говоря, выбирается неоднозначно.

Потребуем, чтобы многочлен $\Phi(x)$ удовлетворял ряду дополнительных условий. Всюду далее для любого целого числа $c$ параметр $\rho_{2^{n-1}}(c)=\rho(c)$ определяется условиями

$$
\rho(c) \in\left\{1,2, \ldots, 2^{n}-1\right\}, \quad \rho(c) \equiv c \quad\left(\bmod 2^{n}-1\right) .
$$

Заметим, что если $2^{n}-1 \mid c$, то $\rho(c)=2^{n}-1$, в противном случае $\rho(c)$ есть остаток от деления $c$ на $2^{n}-1$.

Для любого $k \in\left\{1, \ldots, 2^{n}-1\right\}$ обозначим через $\mu_{n}(k)=\mu(k)$ наименьшее число из циклотомического класса (см. [9]), которому принадлежит $k$ :

$$
\mu(k)=\min \left\{k, \rho(k 2), \ldots, \rho\left(k 2^{n-1}\right)\right\} .
$$

Пусть $M_{n}=M$ - набор минимальных представителей всех различных циклотомических классов, на которые разбивается множество $\left\{1, \ldots, 2^{n}-1\right\}$, то есть

$$
M=\left\{\mu(k): k \in\left\{1, \ldots, 2^{n}-1\right\}\right\} .
$$

Подмножество $M \subset\left\{1, \ldots, 2^{n}-1\right\}$ можно охарактеризовать также следующими условиями:

(а) для любого $s \in\left\{1, \ldots, 2^{n}-1\right\}$ существуют $t \in M$ и $k \in\{0,1, \ldots, n-1\}$ такие, что

$$
s=\rho\left(t 2^{k}\right)
$$


(б) для любых различных $s, t \in M$ и любого $k \in\left\{1, \ldots, 2^{n}-1\right\}$

$$
s \neq \rho\left(t 2^{k}\right)
$$

(в) если числа $s, t \in\left\{1, \ldots, 2^{n}-1\right\}$ при некотором значении $k \in\{1, \ldots, n-1\}$ удовлетворяют условию $s=\rho\left(t 2^{k}\right)$ и $t \in M$, то $s>t$.

Тогда, с учетом свойства $\operatorname{tr}(x)=\operatorname{tr}\left(x^{2}\right)$, можно утверждать, что в представлении (2.3) многочлен $\Phi(x)$ может быть выбран в виде

$$
\Phi(x)=\sum_{k \in M} a_{k} x^{k}, \quad a_{k} \in Q, \quad k \in M,
$$

однако, если $n$ не является простым числом, и такое представление функции $F$ не однозначно.

Зафиксируем примитивный элемент $\theta$ поля $Q$. Каждому числу $k \in M$ поставим в соответствие подполе $Q_{k}=P\left(\theta^{k}\right)$ поля $Q$. Заметим, что $Q_{k}=G F\left(2^{r}\right)$, где

$$
r=r(k)=\min \left\{t \in \mathbf{N}:\left(2^{n}-1\right) /\left(2^{n}-1, k\right) \mid\left(2^{t}-1\right)\right\} .
$$

Пусть

$$
\operatorname{tr}_{O_{k}}^{Q}(x)=\sum_{j=0}^{n / r-1} x^{2^{r j}}
$$

- след из поля $Q$ в подполе $Q_{k}$. Для дальнейшего изложения результатов выберем и зафиксируем систему элементов

$$
\xi_{k} \in Q, \quad \operatorname{tr}_{O_{k}}^{Q}\left(\xi_{k}\right)=e, \quad k \in M
$$

Тогда (см. [10]) существует представление (2.3) функции $F$, в котором многочлен $\Phi(x)$ имеет вид

$$
\Phi(x)=\sum_{k \in M} c_{k} \xi_{k} x^{k}, \quad c_{k} \in Q_{k}, \quad k \in M .
$$

В дальнейшем многочлен $\Phi(x)$ вида (2.5) будем называть редуцированным многочленом, а представление $F(x)=\operatorname{tr}(\Phi(x))$, где многочлен $\Phi(x)$ имеет вид $(2.5),-$ приведенным представлением для $F(x)$.

Для редуцированного многочлена $\Phi(x)$ определим индекс нелинейности ind $\Phi(x)$ paвенством

$$
\text { ind } \Phi(x)=\max \left\{\|k\|: k \in M c_{k} \neq 0\right\},
$$

где $\|k\|-$ двоичный вес числа $k$, то есть число ненулевых цифр в записи числа $k$ в двоичной системе счисления.

С'тепень нелинейности булевой функщии $f\left(x_{0}, \ldots, x_{n-1}\right)$ обозначим через $\operatorname{deg} f$.

Теперь результат из [10] о существовании приведенного представления можно дополнить следующим образом.

Теорема 1. Для любой булевой функции $f\left(x_{0}, x_{1}, \ldots, x_{n-1}\right)=F(x)$ от $n$ переменных существует единственное приведенное представление (2.3)-(2.5) над полем $Q=G F\left(2^{n}\right)$. При этом $\operatorname{deg} f\left(x_{0}, x_{1}, \ldots, x_{n-1}\right)=$ ind $\Phi(x)$. 
Доказательство. Рассмотрим двоичную последовательность $и$ элементов

$$
u(i)=F\left(\theta^{i}\right)
$$

Это реверсивная (периодическая без подхода) последовательность, период которой делит $2^{n}-1=\operatorname{ord} \theta$. Следовательно, $u$ однозначно раскладывается в сумму линейных рекуррент с неприводимыми характеристическими многочленами, степени которых делят $n$. Для каждой рекурренты $v$ из последней суммы существуют единственные $k \in M$ и $c_{k} \in Q_{k}$ такие, что

$$
v(i)=\operatorname{tr}_{P}^{Q_{k}}\left(c_{k}\left(\theta^{k}\right)^{i}\right)=\operatorname{tr}_{P}^{Q}\left(c_{k} \xi_{k}\left(\theta^{i}\right)^{k}\right) .
$$

Таким образом, последовательность $u$ однозначно представляется в виде

$$
u(i)=F\left(\theta^{i}\right)=\sum_{k \in M} \operatorname{tr}_{P}^{Q}\left(c_{k} \xi_{k}\left(\theta^{i}\right)^{k}\right)
$$

Так как $\theta$ - примитивный элемент поля $Q$, последнее равенство означает, что многочлен (2.5) удовлетворяет равенству (2.3) для всех ненулевых $x \in Q$. Справедливость (2.3) для $x=0$ очевидна.

Заметим теперь, что ввиду (2.2) справедливо равенство

$$
u(i)=f\left(\operatorname{tr}\left(\omega_{0} \theta^{i}\right), \ldots, \operatorname{tr}\left(\omega_{n-1} \theta^{i}\right)\right),
$$

причем каждый из аргументов $\operatorname{tr}\left(\omega_{s} \theta^{i}\right)$ в правой части (2.7) есть $i$-й знак линейной рекурренты максимального периода над полем $P$ с характеристическим многочленом, равным минимальному многочлену элемента $\theta$ над полем $P$ (см., например, теорему 10 гл. XXV в т. 2 в [6]). Из результатов [2] следует, что совокупность последовательностей вида (2.7), соответствующих множеству функций $f$, удовлетворяющих условию $\operatorname{deg} f \leqslant t$, совпадает с совокупностью последовательностей вида (2.6), удовлетворяющих условию $\|k\| \leqslant t$, если $c_{k} \neq 0$. Ввиду сказанного выше последнее означает, что ind $\Phi(x)=\operatorname{deg} f$.

\section{3. Мономиальные аналоги и гипер-бент функции}

Функцию $L_{a}^{(\delta)}: Q \rightarrow P$ вида

$$
L_{a}^{(\delta)}(x)=\operatorname{tr}\left(a x^{\delta}\right), \quad a \in Q, \quad \delta \in M,
$$

следуя [5], назовем мономиальной (это функщия, у которой в приведенном представлении (2.3) многочлен $\Phi$ является мономом). Представляется интересным оценить возможности приближения произвольной функции $F$ функциями из класса $\mu$ всех мономиальных функций на $Q$. В данной работе мы ограничимся изучением с этой точки зрения лишь класса $\mu^{*}$ всех собственных мономиальных функций, так мы называем функции (3.1), удовлетворяющие дополнительному условию $\left(\delta, 2^{n}-1\right)=1$.

Заметим, что любая линейная функция есть собственная мономиальная функция, так как для каждого $a \in Q$ справедливо равенство $L_{a}(x)=L_{a}^{(1)}(x)$. Таким образом,

$$
\mathscr{L} \subset M^{*}
$$

и известные оценки вероятности приближения произвольной булевой функции линейными статистическими аналогами являются оценками снизу для вероятности приближения булевой функции мономиальными аналогами. 
В обозначениях (1.1), (1.2), (1.3) представляется интересным

- оценить (вычислить) $\Delta \mathcal{M}^{*}$;

- описать функции $F$, удовлетворяющие как условию

$$
\Delta\left(F, \mathcal{M}^{*}\right)=\Delta\left(\mathcal{M}^{*}\right),
$$

так и условию

$$
\Delta\left(F, M^{*}\right)>\Delta\left(M^{*}\right)
$$

- найти для данной функции $F$ наилучший собственный мономиальный аналог, то есть функцию $L_{a}^{(\delta)} \in M^{*}$, удовлетворяющую условию

$$
\Delta\left(F, L_{a}^{(\delta)}\right)=\Delta\left(F, M^{*}\right)
$$

Мы по-прежнему считаем, что $n=2 \lambda-$ четное число. Следовательно, верно (1.4). Отсюда и из (3.2) следует неравенство

$$
\Delta \mu^{*} \geqslant 2^{\lambda}
$$

В [12] доказано, что, в действительности,

$$
\Delta M^{*}=\Delta \mathscr{L}=2^{\lambda},
$$

и построен пример функции $F$, удовлетворяющей равенству

$$
\Delta\left(F, \mu^{*}\right)=2^{\lambda} .
$$

Следуя [12], мы будем называть функщии, удовлетворяющие последнему равенству, гипербент-функциями. Из определения и предложения 1 видно, что любая гипер-бент-функция есть бент-функция.

Цель этой работы - описать ряд ограничений на редуцированный многочлен $\Phi(x)$ в представлении (2.3) гипер-бент-функции $F$ и показать, что класс гипер-бент-функций существенно меньше класса бент-функций. Следовательно, существуют бент-функции $F$, для которых

$$
\Delta\left(F, \mu^{*}\right)>\Delta(F, \mathscr{L})=2^{\lambda} .
$$

Для формулировки основного результата обозначим через $M(\lambda)$ подмножество всех чисел $k \in M$, удовлетворяющих условию

$$
\forall \sigma \in\left\{k \in\left\{1, \ldots, 2^{n}-1\right\}:\left(k, 2^{n}-1\right)=1\right\}:\|\rho(k \sigma)\|=\lambda .
$$

Нашим основным результатом является следующая теорема.

Теорема 2. Если при введенных выше обозначениях $F: Q \rightarrow P$ есть гипер-бент-функция, то в ее приведенном представлении (2.3)-(2.5) редучированный многочлен (2.5) имеет вид

$$
\Phi(x)=\sum_{k \in M(\lambda)} c_{k} \xi_{k} x^{k}, \quad c_{k} \in Q_{k}, \quad k \in M(\lambda) .
$$

В частности, любая гипер-бент-функчия есть бент-функция степени нелинейности $\lambda$. Для любого $\sigma \in\left\{k \in\left\{1, \ldots, 2^{n}-1\right\}:\left(k, 2^{n}-1\right)=1\right\}$ функиия $F_{\sigma}(x)=F\left(x^{\sigma}\right)$ есть также гипер-бент-функиия. 
Доказательство разобьем на ряд этапов.

Лемма 1. Пусть $a \in Q, \sigma, \delta \in\left\{k \in\left\{1, \ldots, 2^{n}-1\right\}:\left(k, 2^{n}-1\right)=1\right\} u \tau=\rho(\sigma \delta)$. Тогдa

$$
\Delta\left(F, L_{a}^{(\delta)}\right)=\Delta\left(F_{\sigma}, L_{a}^{(\tau)}\right)
$$

где $F_{\sigma}(x)=F\left(x^{\sigma}\right)$. В частности, если $\tau=1$, то

$$
\Delta\left(F, L_{a}^{(\delta)}\right)=\Delta\left(F_{\sigma}, L_{a}\right) .
$$

Доказательство. Из определений следует, что

$$
\Delta\left(F, L_{a}^{(\delta)}\right)=\sum_{x \in Q}(-1)^{F(x)+\operatorname{tr}\left(a x^{\delta}\right)} .
$$

Проведя в этом равенстве замену переменных $x=y^{\sigma}$ и пользуясь равенством функций $\operatorname{tr}\left(y^{\sigma \delta}\right)=\operatorname{tr}\left(y^{\tau}\right)$ на $Q$, получаем, что

$$
\Delta\left(F, L_{a}^{(\delta)}\right)=\sum_{y \in Q}(-1)^{F\left(y^{\sigma}\right)+\operatorname{tr}\left(a y^{\tau}\right)}=\Delta\left(F_{\sigma}, L_{a}^{(\tau)}\right) .
$$

В частности, отсюда следует, что при фиксированном значении $\delta$ набор характеристик $\Delta\left(F, L_{a}^{(\delta)}\right), a \in Q$, для функции $F(x)$ совпадает с набором характеристик $\Delta\left(F_{\sigma}, L_{a}\right)$, $a \in Q$, для функщии $F_{\sigma}$, где $\rho(\sigma \delta)=1$, и изучение собственных мономиальных аналогов для функции $F(x)$ можно свести к изучению линейных аналогов для семейства функций $F_{\sigma}(x), \sigma \in\left\{k \in\left\{1, \ldots, 2^{n}-1\right\}:\left(k, 2^{n}-1\right)=1\right\}$.

Следующее предложение аналогично предложению 1.

Предложение 2. Для функчии $F$ следующие условия эквивалентны:

(a) F- гипер-бент-функиия;

(б) $\left|\Delta\left(F, L_{a}^{(\delta)}\right)\right| \leqslant 2^{\lambda}$ для всех $a \in Q, \delta \in\left\{k \in\left\{1, \ldots, 2^{n}-1\right\}:\left(k, 2^{n}-1\right)=1\right\}$;

(в) $\left|\Delta\left(F, L_{a}^{(\delta)}\right)\right|=2^{\lambda}$ для всех $a \in Q, \delta \in\left\{k \in\left\{1, \ldots, 2^{n}-1\right\}:\left(k, 2^{n}-1\right)=1\right\}$;

(г) $F_{\sigma}-$ бент-функция для каждого $\sigma \in\left\{k \in\left\{1, \ldots, 2^{n}-1\right\}:\left(k, 2^{n}-1\right)=1\right\}$;

(д) $F_{\sigma}$ - гипер-бент-функция для каждого $\sigma \in\left\{k \in\left\{1, \ldots, 2^{n}-1\right\}:\left(k, 2^{n}-1\right)=1\right\}$.

Доказательство. Импликация (а) $\Rightarrow$ (б) есть следствие определений (1.2) и (3.4).

Импликация (б) $\Rightarrow$ (г) следует из леммы 1 и пунктов (б) и (в) предложения 1.

Импликация (г) $\Rightarrow$ (в) следует из леммы 1 и пунктов (в) и (а) предложения 1.

Импликация (в) $\Rightarrow$ (а) очевидна.

Наконец, согласно лемме 1 , совокупность параметров $\Delta\left(F, L_{a}^{(\delta)}\right), a \in Q$, $\delta \in\left\{k \in\left\{1, \ldots, 2^{n}-1\right\}:\left(k, 2^{n}-1\right)=1\right\}$, совпадает с совокупностью параметров $\Delta\left(F_{\sigma}, L_{a}^{(\tau)}\right), a \in Q, \tau \in\left\{k \in\left\{1, \ldots, 2^{n}-1\right\}:\left(k, 2^{n}-1\right)=1\right\}$. Отсюда следует эквивалентность (в) и (д).

Предложение 3. Если $F$ - гипер-бент-функция, то многочлен (2.5) из (2.3) имеет вид

$$
\Phi(x)=\sum_{k \in M,\|k\|=\lambda} c_{k} \xi_{k} x^{k}
$$


Доказательство. Так как по предложению $2 F$ есть бент-функция, по теореме 1 и предложению 1 справедливо неравенство ind $\Phi \leqslant \lambda$. Следовательно, в (2.5) могут быть отличны от нуля лишь коэффициенты $a_{k}$ с номерами $k$, удовлетворяющими условию $\|k\| \leqslant \lambda$.

Покажем теперь, что если существует $k \in M$ такое, что

$$
\|k\|=t<\lambda, \quad c_{k} \neq 0
$$

то для некоторого $\sigma \in\left\{k \in\left\{1, \ldots, 2^{n}-1\right\}:\left(k, 2^{n}-1\right)=1\right\}$ приведенный многочлен $\Phi_{\sigma}(x)$ из представления

$$
F_{\sigma}(x)=F\left(x^{\sigma}\right)=\operatorname{tr}\left(\Phi_{\sigma}(x)\right)
$$

удовлетворяет условию

$$
\text { ind } \Phi_{\sigma}>\lambda
$$

что противоречит пунктам (а) и (г) предложения 2.

Из (2.3) следует, что функции $F_{\sigma}$ удовлетворяет равенству

$$
F_{\sigma}(x)=\operatorname{tr}\left(\sum_{k \in M} c_{k} \xi_{k} x^{k^{\prime}}\right)
$$

где $k^{\prime}=\rho(k \sigma)$. Однако полином, стоящий в правой части этого равенства, в общем случае не является приведенным. Для получения приведенного многочлена, представляющего $F_{\sigma}$ необходимо в (3.10) каждую из степеней $k^{\prime}$ заменить числом $k^{\prime \prime}=\mu\left(k^{\prime}\right)$. При этом $k^{\prime \prime}=\rho\left(k^{\prime} 2^{l_{k}}\right)$ для некоторого $l_{k} \in\{0,1, \ldots, n-1\}$ и справедливо равенство

$$
F_{\sigma}(x)=\operatorname{tr}\left(\sum_{k \in M} \bar{c}_{k^{\prime \prime}} \bar{\xi}_{k^{\prime \prime}} x^{k^{\prime \prime}}\right),
$$

где $\bar{c}_{k^{\prime \prime}}=c_{k}^{2^{l} k}, \bar{\xi}_{k^{\prime \prime}}=\xi_{k}^{2^{l} k}$. Отсюда, пользуясь определением элемента $\xi_{k^{\prime \prime}}$, получаем также равенство

$$
F_{\sigma}(x)=\operatorname{tr}\left(\sum_{k \in M} \bar{c}_{k^{\prime \prime}} \xi_{k^{\prime \prime}} x^{k^{\prime \prime}}\right)
$$

Нетрудно проверить, что по определению множества $M$ для различных $k_{1}, k_{2} \in M$ и любых $l_{1}, l_{2} \in\{0,1, \ldots, n-1\}$ числа $\rho\left(k_{1} \sigma 2^{l_{1}}\right)$ и $\rho\left(k_{2} \sigma 2^{l_{2}}\right)$ принадлежат различным циклотомическим классам, и следовательно, соответствующие числа $k_{1}^{\prime \prime}, k_{2}^{\prime \prime}$ суть различные числа из $M$. Другими словами, отображение $g_{\sigma}: M \rightarrow M$ по правилу $g_{\sigma}(k)=k^{\prime \prime}=\mu(\rho(k \sigma))$ есть подстановка на $M$. Теперь, полагая $h_{\sigma}=g_{\sigma}^{-1}$, из последнего равенства получаем, что приведенный многочлен, представляющий $F_{\sigma}$, есть

$$
\Phi_{\sigma}(x)=\sum_{k^{\prime \prime} \in M} \bar{c}_{k^{\prime \prime}} \xi_{k^{\prime \prime}} x^{k^{\prime \prime}}=\sum_{k \in M} c_{k}^{(\sigma)} \xi_{k} x^{k}
$$

где $c_{k}^{(\sigma)}=c_{h_{\sigma}(k)}^{2^{l} h_{\sigma(k)}}$. При этом

$$
\forall k \in M:\left(\bar{c}_{k^{\prime \prime}} \neq 0 \Longleftrightarrow c_{k} \neq 0\right) \&\left\|k^{\prime \prime}\right\|=\|\rho(k \sigma)\| .
$$


Следовательно, число ненулевых слагаемых в многочлене $\Phi_{\sigma}(x)$ останется таким же, как в многочлене $\Phi(x)$, и справедливо равенство

$$
\text { ind } \Phi_{\sigma}(x)=\max \left\{\|\rho(k \sigma)\|: k \in M, c_{k} \neq 0\right\} \text {. }
$$

Теперь при условии (3.7) положим $\sigma=2^{n}-2$. Очевидно, что $\left(\sigma, 2^{n}-1\right)=1$ и $k \sigma \equiv-k \equiv 2^{n}-1-k\left(\bmod 2^{n}-1\right)$. Следовательно,

$$
\rho(k \sigma)=2^{n}-1-k, \quad\|\rho(k \sigma)\|=n-t>\lambda .
$$

Таким образом, ввиду (3.13) для выбранного $\sigma$ выполняется условие (3.9).

Доказательство теоремы 2. Для завершения доказательства теоремы 2 рассмотрим носитель $S(\Phi)$ многочлена $\Phi(x)$, определяемый при условии (2.5) равенством

$$
S(\Phi)=\left\{k \in M: c_{k} \neq 0\right\} .
$$

Согласно (3.12) для каждого $\sigma \in\left\{k \in\left\{1, \ldots, 2^{n}-1\right\}:\left(k, 2^{n}-1\right)=1\right\}$ в обозначениях из доказательства предложения 3 справедливо равенство

$$
S\left(\Phi_{\sigma}\right)=\left\{k^{\prime \prime} \in M: k \in S(\Phi)\right\} .
$$

Согласно пунктам (а) и (д) предложения 2 каждая из функщий $F_{\sigma}$ есть гипер-бентфункция, и следовательно, по предложению 3 каждое $k^{\prime \prime} \in S\left(\Phi_{\sigma}\right)$ удовлетворяет условию $\left\|k^{\prime \prime}\right\|=\lambda$. Последнее условие равносильно условию $\|\rho(k \sigma)\|=\lambda$, так как $k^{\prime \prime}=\rho\left(k \sigma 2^{l_{k}}\right)$

Итак, если $k \in S(\Phi)$, то $\|\rho(k \sigma)\|=\lambda$ для $\sigma \in\left\{k \in\left\{1, \ldots, 2^{n}-1\right\}:\left(k, 2^{n}-1\right)=1\right\}$, то есть $S(\Phi) \subseteq M(\lambda)$.

Теорема 2 доказана.

В связи с полученным результатом представляет интерес чисто арифметическая задача описания множества $M(\lambda)$ или оценки его мощности. В этом направлении можно предложить следующие результаты.

Предложение 4. Для любого $n=2 \lambda>2$ и любого натурального делителя $s$ числа $\lambda$ справедливо включение

$$
\frac{2^{n}-1}{2^{s}+1} \in M(\lambda)
$$

в частности,

$$
\frac{2^{n}-1}{3} \in M(\lambda), \quad 2^{\lambda}-1 \in M(\lambda) .
$$

Для любого $а \in\left\{1, \ldots, 2^{\lambda}\right\}$ выполняется включение

$$
\mu\left(\left(2^{\lambda}-1\right) a\right) \in M(\lambda) .
$$

$\Pi$ Путь $t \in \mathbf{N} \backslash\{1\}, m=1+2^{n}+\ldots+2^{(t-1) n}$. Тогда

$$
m M(\lambda) \subseteq M(t \lambda)
$$

Сначала докажем вспомогательное утверждение. 
Лемма 2. Пусть $a \in\left\{1, \ldots, 2^{s}\right\}$. Тогда число $а\left(2^{s}-1\right)$ имеет двоичный вес

$$
\left\|a\left(2^{s}-1\right)\right\|=s .
$$

Доказательство. Для случая, когда $а$ нечетно, это утверждение есть в точности лемма 4 из [12]. Если же $a=2^{t} a_{1}$, где $a_{1}$ нечетно, то достаточно заметить, что по упомянутой лемме справедливо равенство $\left\|a_{1}\left(2^{s}-1\right)\right\|=2^{s}$, а двоичное представление числа $a\left(2^{s}-1\right)$ получается сдвигом на $t$ разрядов двоичного представления числа $a_{1}\left(2^{s}-1\right)$.

Доказательство предложения 4. Докажем первое утверждение. Пусть $\lambda=s t$, тогда $2^{n}-1=\left(2^{2 s}-1\right)\left(1+2^{2 s}+\ldots+2^{2 s(t-1)}\right)$ и число $k=\left(2^{n}-1\right) /\left(2^{s}+1\right)$ имеет вид

$$
k=\left(2^{s}-1\right)+2^{2 s}\left(2^{s}-1\right)+\ldots+2^{2 s(t-1)}\left(2^{s}-1\right) .
$$

Очевидно, что двоичное разложение этого числа содержит ровно $s t=\lambda$ разрядов, равных единице, то есть $\|k\|=\lambda$. Пусть $\sigma \in\left\{k \in\left\{1, \ldots, 2^{n}-1\right\}:\left(k, 2^{n}-1\right)=1\right\}$ и $a-$ остаток от деления $\sigma$ на $2^{s}+1$. Тогда $\sigma=d\left(2^{s}+1\right)+a, k \sigma=d\left(2^{n}-1\right)+k a$, откуда $k \sigma \equiv k a\left(\left(\bmod 2^{n}-1\right)\right)$, и так как $1 \leqslant k a \leqslant 2^{n}-2$, то $\rho(k \sigma)=k a$.

Из (3.17) следует, что

$$
k a=\left(2^{s}-1\right) a+2^{2 s}\left(2^{s}-1\right) a+\ldots+2^{2 s(t-1)}\left(2^{s}-1\right) a .
$$

Так как $1 \leqslant\left(2^{s}-1\right) a \leqslant 2^{2 s}-2$, из (3.18) следует, что $n$-разрядное двоичное представление числа $k a$ получается $t$-кратным повторением $2 s$-разрядного двоичного представления числа $\left(2^{s}-1\right) a$. Теперь, используя лемму 2 , получаем, что

$$
\|\rho(k \sigma)\|=\|k a\|=t\left\|\left(2^{s}-1\right) a\right\|=t s=\lambda .
$$

Докажем второе утверждение. С учетом леммы 2 достаточно доказать, что для любого $\sigma \in\left\{k \in\left\{1, \ldots, 2^{n}-1\right\}:\left(k, 2^{n}-1\right)=1\right\}$ справедливо равенство

$$
\mid \rho\left(\left(2^{\lambda}-1\right) a \sigma\right) \|=\lambda \text {. }
$$

Так как $2^{\lambda}-1 \mid 2^{n}-1$, то $\rho\left(\left(2^{\lambda}-1\right) a \sigma\right)=\left(2^{\lambda}-1\right) b$, где $b-$ остаток от деления $a \sigma$ на $2^{\lambda}+1$. Теперь (3.19) следует из леммы 2 .

Докажем третье утверждение. По определению включение $k \in M(\lambda)$ равносильно выполнению условия (3.5). Для доказательства (3.16) покажем сначала, что

$$
\forall \tau \in\left\{k \in\left\{1, \ldots, 2^{n}-1\right\}:\left(k, 2^{n}-1\right)=1\right\}:\left\|\rho_{2^{t n-1}}(m k \tau)\right\|=t \lambda .
$$

Так как $2^{t n}-1=m\left(2^{n}-1\right)$, то

$$
\rho_{2^{t n-1}}(m k \tau)=m \rho_{2^{n-1}}(k \tau)=m \rho_{2^{n-1}}\left(k \rho_{2^{n}-1}(\tau)\right) .
$$

Так как $\rho_{2^{n}-1}(\tau) \in\left\{k \in\left\{1, \ldots, 2^{n}-1\right\}:\left(k, 2^{n}-1\right)=1\right\}$, ввиду (3.5) число $a=$ $\rho_{2^{n-1}}\left(k \rho_{2^{n}-1}(\tau)\right)$ имеет норму $\|a\|=\lambda$, и так как $a<2^{n}$, то

$$
\left\|\rho_{2^{t n-1}}(m k \tau)\right\|=\|m a\|=\left\|a+2^{n} a+\ldots+2^{(t-1) n} a\right\|=t\|a\|=t \lambda,
$$

то есть верно (3.20). 
Теперь остается доказать, что $m k$ - наименьшее число в циклотомическом классе $\left\{m k, \rho_{2^{t n-1}}(m k 2), \ldots, \rho_{2^{t n-1}}\left(m k 2^{t n-1}\right)\right\}$. Каждое из чисел $K=\rho_{2^{t n-1}}\left(m k 2^{s}\right)$ имеет вид

$$
K=m \rho_{2^{n-1}}\left(k \rho_{2^{n-1}}\left(2^{s}\right)\right)=m \rho_{2^{n-1}}\left(k 2^{r}\right),
$$

где $r$ - остаток от деления $s$ на $n$. По определению множества $M$ справедливо неравенство $k<\rho_{2^{n-1}}\left(k 2^{r}\right)$, поэтому $m k<K$.

Все три утверждения предложения 4 доказаны.

Будем называть элементы $k, l \in M(\lambda)$ ассоциированными и писать $k \sim l$, если $l=\rho(k \sigma)$ для некоторого $\sigma \in\left\{k \in\left\{1, \ldots, 2^{n}-1\right\}:\left(k, 2^{n}-1\right)=1\right\}$. Очевидно, что $\sim$ есть отношение эквивалентности на $M(\lambda)$. Обозначим через $[k]$ класс чисел из $M(\lambda)$, ассоциированных $\mathrm{c} k$.

Предложение 5. Пусть $k \in M(\lambda) u d=\left(k, 2^{n}-1\right)$. Тогда $d \in[k] u d-$ единственный делитель числа $2^{n}-1$, принадлежащий классу $[k]$.

Доказательство. Рассмотрим кольцо $R=\mathrm{Z}_{2^{n-1}}$ с операцией умножения, обозначаемой точкой. По условию имеет место равенство идеалов

$$
R \cdot k=R \cdot d .
$$

По теореме 20.9 в [7] существует обратимый элемент $\sigma \in R^{*}$ такой, что $d=k \cdot \sigma$. Так как $d \neq 0$, последнее равенство дает равенство $d=\rho(k \sigma)$. Следовательно, $d \in[k]$. Если $d^{\prime} \in[k]$ и $\left.d^{\prime}\right|_{\mathrm{z}} 2^{n}-1$, то $R \cdot k=R \cdot d^{\prime}$ и $d=d^{\prime}$, так как идеал $R \cdot k$ содержит единственный образующий, делящий $2^{n}-1$, именно, наименьший ненулевой элемент из $R \cdot k$.

Следствие 1. Пусть $D(\lambda)-$ множество чисел $d \in M(\lambda)$, делящих $2^{n}-1$. Тогда

$$
M(\lambda)=\bigcup_{d \in D(\lambda)}[d]
$$

где $[d]$ - класс чисел из множества $M(\lambda)$, ассочиированных с $d$.

Таким образом, для перечисления всех элементов множества $M(\lambda)$ достаточно перечислить лишь те из них, которые делят $2^{n}-1$, то есть составляют множество $D(\lambda)$. Затем для каждого $d \in D(\lambda)$, следует вычислить все числа $\rho(d \sigma), \sigma \in\left\{k \in\left\{1, \ldots, 2^{n}-1\right\}\right.$ : $\left.\left(k, 2^{n}-1\right)=1\right\}$. Множество этих чисел есть, очевидно, объединение циклотомических классов, и каждое из этих чисел по определению $M(\lambda)$ имеет норму $\lambda$. Множество минимальных элементов из указанных циклотомических классов и составляет множество $[d]$ из формулировки следствия 1.

Компьютерные вычисления для $\lambda \in\{2,3, \ldots, 30\}$ дали результаты, приведенные ниже в табл. 1 , где символом * помечены те элементы множества $M(\lambda)$, существование которых не вытекает из предложения 4.

\section{4. Один класс гипер-бент-функций}

Прежде всего приведем результат из [12], описывающий единственный известный до сих пор класс гипер-бент-функщий. Наши формулировка и доказательство этого результата представляются более короткими.

Заметим, что мультипликативная группа поля $Q=G F\left(2^{n}\right)$ с единицей $e$ есть прямое произведение $Q^{*}=A \dot{\times} B$, где $A$ есть мультипликативная группа поля $K=G F\left(2^{\lambda}\right), B-$ группа порядка $2^{\lambda}+1$. 
Теорема 3. Пусть $G: B \cup\{0\} \rightarrow P-$ функиия, принимающая значение е ровно $t$ раз $и$ $G(0)=0$. Тогда функция

$$
F(x)=G\left(x^{2^{\lambda}-1}\right)
$$

на $Q$ есть гипер-бент-функция, если и только если $t=2^{\lambda-1}$.

Докажем вспомогательное утверждение.

Лемма 3. В обозначениях (1.1) справедливы соотношения

$$
\Delta(F, \mathrm{tr})= \begin{cases}2 t, & \text { если } G(e)=0 \\ -2^{\lambda+1}+2 t, & \text { если } G(e)=1\end{cases}
$$

Доказательство. Пользуясь определением и разложением $Q^{*}=A \dot{\times} B$ получаем, что

$$
\begin{aligned}
\Delta(F, \operatorname{tr}) & =\sum_{x \in Q}(-1)^{F(x)+\operatorname{tr}(x)}=1+\sum_{x \in Q^{*}}(-1)^{F(x)+\operatorname{tr}(x)}=1+\sum_{y \in A} \sum_{z \in B}(-1)^{F(y z)+\operatorname{tr}(y z)} \\
& =1+\sum_{y \in A} \sum_{z \in B}(-1)^{G\left(z^{2^{\lambda}-1}\right)+\operatorname{tr}_{P}^{Q}(y z)}=1+\sum_{z \in B}(-1)^{G\left(z^{-2}\right)} \sum_{y \in A}(-1)^{\operatorname{tr}_{P}^{K}\left(\operatorname{tr}_{K}^{Q}(z) y\right)} \\
K & =G F\left(2^{\lambda}\right) .
\end{aligned}
$$

Заметим, что для любого $z \in B$ справедливо равенство

$$
\operatorname{tr}_{K}^{Q}(z)=z+z^{2^{\lambda}}=z+z^{-1},
$$

так как $z^{2^{\lambda}+1}=1$. В частности, $\operatorname{tr}_{K}^{Q}(1)=0$, и если $z \in B \backslash\{1\}$, то $\operatorname{tr}_{K}^{Q}(z) \neq 0$, так как иначе $z^{-1}=z$ и $z^{2}=1$, что невозможно.

Таким образом, сумма $\sum_{y \in A}(-1)^{\operatorname{tr}_{P}^{K}\left(\operatorname{tr}_{K}^{Q}(z) y\right)}$ равна $2^{\lambda}-1$, если $z=e$, и, ввиду условия $A=K^{*}$, равна -1 , если $z \in K \backslash\{e\}$. Отсюда

$$
\Delta(F, \operatorname{tr})=1+(-1)^{G(e)}\left(2^{\lambda}-1\right)-\sum_{z \in B \backslash\{e\}}(-1)^{G(z)} .
$$

Из последнего равенства следует, что если $G(e)=0$, то

$$
\begin{gathered}
\sum_{z \in B \backslash\{e\}}(-1)^{G(z)}=|B|-1-2 t=2^{\lambda}-2 t, \\
\Delta(F, \operatorname{tr})=1+\left(2^{\lambda}-1\right)-\left(2^{\lambda}-2 t\right)=2 t,
\end{gathered}
$$

если же $G(e)=1$, то

$$
\begin{gathered}
\sum_{z \in B \backslash\{e\}}(-1)^{G(z)}=|B|-1-2(t-1)=2^{\lambda}-2 t+1, \\
\Delta(F, \text { tr })=1-\left(2^{\lambda}-1\right)-\left(2^{\lambda}-2 t+1\right)=-2^{\lambda+1}+2 t .
\end{gathered}
$$

Доказательство теоремы 3. Заметим теперь, что равенство

$$
\Delta\left(F(x), \operatorname{tr}\left(\xi x^{\delta}\right)\right)=\Delta\left(F^{\prime}(x), \operatorname{tr}(x)\right)
$$


справедливо для любых $\xi \in Q^{*}, \delta \in\left\{k \in\left\{1, \ldots, 2^{n}-1\right\}:\left(k, 2^{n}-1\right)=1\right\}$ где $F^{\prime}(x)=$ $F\left(\sigma x^{\mu}\right), \sigma=\xi^{-1}, \mu \in\left\{k \in\left\{1, \ldots, 2^{n}-1\right\}:\left(k, 2^{n}-1\right)=1\right\}, \rho(\mu \delta)=1$. При этом $F^{\prime}(x)=G^{\prime}\left(x^{2^{\lambda}-1}\right)$, где $G^{\prime}(x)=G\left(\sigma^{2^{\lambda}-1} x^{\mu}\right)$. Ясно, что соответствие $x \rightarrow \sigma^{2^{\lambda}-1} x^{\mu}$ задает подстановку на $B \cup\{0\}$ и функция $G^{\prime}: B \cup\{0\} \rightarrow P$, так же, как и $G$, принимает значение $e$ ровно $t$ раз. Таким образом, ввиду равенства (4.2) и леммы 3 функция $F$ из условия теоремы удовлетворяет следующему условию: для любых $\xi \in Q^{*}$, $\delta \in\left\{k \in\left\{1, \ldots, 2^{n}-1\right\}:\left(k, 2^{n}-1\right)=1\right\}$ справедливо включение $\Delta\left(F(x), \operatorname{tr}\left(\xi x^{\delta}\right)\right) \in$ $\left\{2 t,-2^{\lambda+1}+2 t\right\}$ и $F$ есть гипер-бент-функция в точности если $t=2^{\lambda-1}$.

Для некоторых значений $n$ гипер-бент-функции, описанные в последней теореме, исчерпывают весь класс гипер-бент-функциий.

Теорема 4. Пусть все числа множества $M(\lambda)$ делятся на $2^{\lambda}-1$. Тогда в обозначениях теоремы 3 произвольная функция $F: Q \rightarrow P$ со свойством $F(0)=0$ есть гипер-бентфункчия в точности если она имеет вид

$$
F(x)=G\left(x^{2^{\lambda}-1}\right),
$$

где

$$
G: B \cup\{0\} \rightarrow P, \quad G(0)=0,
$$

и $G$ принимает на В значение е ровно $2^{\lambda-1}$ раз.

Доказательство. По условию каждый элемент $k \in M(\lambda)$ имеет вид $k=k_{1}\left(2^{\lambda}-1\right)$. Тогда по формуле (3.6) многочлен $\Phi(x)$ из (2.3) имеет вид

$$
\Phi(x)=\sum_{k \in M(\lambda)} c_{k} \xi_{k}\left(x^{2^{\lambda}-1}\right)^{k_{1}}
$$

Отсюда для $F$ получается нужное выражение (4.3). Остается заметить, что по теореме 3 сформулированные условия на функцию (4.4) являются необходимыми и достаточными для того, чтобы функция $F$ была гипер-бент-функцией.

Следующий результат описывает некоторое множество значений параметра $n$, удовлетворяющих условию теоремы 4.

Предложение 6. Пусть $n=2 \lambda>4 u p=2^{\lambda}-1$ - простое число, тогда все элементы множества $M(\lambda)$ делятся на р и все гипер-бент-функции от $n$ аргументов описываются теоремой 4.

Доказательство. Достаточно доказать, что если $d \in M(\lambda)$ и $d \mid 2^{n}-1$, то $p \mid d$, так как любое число $k \in M(\lambda)$, имея по предложению 5 вид $k=\rho(d \sigma)$, делится на $p$.

Если $\|d\|=\lambda>2$ и $d \neq 2^{\lambda}-1$, то $d>2^{\lambda}+1$, поскольку $d \geqslant 2^{\lambda}-1,\left\|2^{\lambda}\right\|=1$, $\left\|2^{\lambda}+1\right\|=2$. Следовательно, $\left(d, 2^{\lambda}-1\right)=(d, p)>1$ и $p \mid d$, так как $p-$ простое число.

В дополнение к предложению 6 следует отметить, что если $n=4$, то, несмотря на то, что $\lambda=2$ и $2^{\lambda}-1=3$ - простое число, множество $M(\lambda)=M(2)$ содержит число 5 , не делящееся на $2^{\lambda}-1$. Более того, компьютерные вычисления показывают, что среди функций со свойством $F(0)=0$, помимо десяти функций, описываемых теоремой 4 , существуют еще 18 гипер-бент-функций. Все гипер-бент-функции для $n=4$ описаны в табл. 2. 
Таблица 1. Множества $D(\lambda)$ для $\lambda=2, \ldots, 30$

\begin{tabular}{|c|c|}
\hline \multicolumn{2}{|c|}{ Делители $d$ числа $2^{n}-1$, принадлежащие $M(\lambda), n=2 \lambda$} \\
\hline$\lambda$ Варианты $d \in D(\lambda)$ & Двоичная запись $d$ \\
\hline $2 \sqrt{3}$ & 0011 \\
\hline & 0101 \\
\hline 37 & 000111 \\
\hline $21=3 \cdot 7$ & 010101 \\
\hline \begin{tabular}{l|l|l}
4 & 15
\end{tabular} & 00001111 \\
\hline 51 & 00110011 \\
\hline $85=5 \cdot 17$ & 01010101 \\
\hline 531 & 0000011111 \\
\hline $93=31 \cdot 3$ & 0001011101 \\
\hline $341=31 \cdot 11$ & 0101010101 \\
\hline$6 \longdiv { 6 3 }$ & 000000111111 \\
\hline $315=63 \cdot 5$ & 000100111011 \\
\hline 455 & 000111000111 \\
\hline $819=63 \cdot 13$ & 001100110011 \\
\hline $1365=21 \cdot 65$ & 010101010101 \\
\hline \begin{tabular}{|l|l|}
7 & 127
\end{tabular} & 00000001111111 \\
\hline $381=127 \cdot 3$ & 00000101111101 \\
\hline $5461=127 \cdot 43$ & 01010101010101 \\
\hline$8 \longdiv { 2 5 5 }$ & 0000000011111111 \\
\hline $3855=15 \cdot 257$ & 0000111100001111 \\
\hline $13107=51 \cdot 257$ & 0011001100110011 \\
\hline $21845=85 \cdot 257$ & 0101010101010101 \\
\hline 9511 & 000000000111111111 \\
\hline $1533=511 \cdot 3$ & 000000010111111101 \\
\hline $4599=511 \cdot 9$ & 000001000111110111 \\
\hline $9709=511 \cdot 19$ & 000010010111101101 \\
\hline $13797=511 \cdot 27$ & 000011010111100101 \\
\hline $29127=511 \cdot 57$ & 000111000111000111 \\
\hline $87381=511 \cdot 171$ & 010101010101010101 \\
\hline 101023 & 00000000001111111111 \\
\hline $5115=1023 \cdot 5$ & 00000001001111111011 \\
\hline $25575=1023 \cdot 25$ & 00000110001111100111 \\
\hline $31775=31 \cdot 1025$ & 00000111110000011111 \\
\hline $41943=1023 \cdot 41$ & 00001010001111010111 \\
\hline 95325 & 00010111010001011101 \\
\hline $209715=1023 \cdot 205$ & 00110011001100110011 \\
\hline $349525=341 \cdot 1025$ & 01010101010101010101 \\
\hline 112047 & 000000000011111111111 \\
\hline $6141=2047 \cdot 3$ & 000000001011111111101 \\
\hline $60787^{*}$ & 0000001110110101110011 \\
\hline $1398101=2047 \cdot 683$ & 0101010101010101010101 \\
\hline 124095 & 000000000000111111111111 \\
\hline $69615=4095 \cdot 17$ & 000000010000111111101111 \\
\hline 258111 & 000000111111000000111111 \\
\hline $986895=4095 \cdot 241$ & 000011110000111100001111 \\
\hline 1290555 & 000100111011000100111011 \\
\hline 1864135 & 000111000111000111000111 \\
\hline 3355443 & 001100110011001100110011 \\
\hline $5592405=1365 \cdot 4097$ & 010101010101010101010101 \\
\hline 1388191 & 00000000000001111111111111 \\
\hline $24573=8191 \cdot 3$ & 00000000000101111111111101 \\
\hline $22369621=8191 \cdot 2731$ & 01010101010101010101010101 \\
\hline$1 4 \longdiv { 1 6 3 8 3 }$ & 0000000000000011111111111111 \\
\hline $81915=16383 \cdot 5$ & 0000000000010011111111111011 \\
\hline $475107=16383 \cdot 29$ & 0000000001110011111111100011 \\
\hline
\end{tabular}




\begin{tabular}{|c|c|}
\hline Делители $d$ числ & $\pi$ \\
\hline$\lambda$ Варианты $d \in D(\lambda)$ & Двоичная запись $d$ \\
\hline $141851279=16383 \cdot 113$ & 0000000111000011111110001111 \\
\hline 2080895 & 0000000111111100000001111111 \\
\hline $2375535=16383 \cdot 145$ & 000000100100001111110110111 \\
\hline 6242685 & 0000010111110100000101111101 \\
\hline $9256395=16383.565$ & 0000100011010011110111001011 \\
\hline $53687091=16383 \cdot 3277$ & 0011001100110011001100110011 \\
\hline $89478485=5461 \cdot 16385$ & 0101010101010101010101010101 \\
\hline$1 5 \longdiv { 3 2 7 6 7 }$ & 000000000000000111111111111111 \\
\hline $98301=32767 \cdot 3$ & 000000000000010111111111111101 \\
\hline $294903=32767 \cdot 9$ & 000000000001000111111111110111 \\
\hline $360437=32767 \cdot 11$ & 00000000000101011111111110101 \\
\hline $1081311=32767 \cdot 33$ & 000000000100000111111111011111 \\
\hline $3243933=32767.99$ & 000000001100010111111110011101 \\
\hline $10845877=32767 \cdot 331$ & 000000101001010111111010110101 \\
\hline 13944699 * & 000000110101001100011101111011 \\
\hline $32537631=32767.993$ & 000001111100000111110000011111 \\
\hline $97612893=32767 \cdot 2979$ & 000101110100010111010001011101 \\
\hline 119304647 & 000111000111000111000111000111 \\
\hline $357913941=32767 \cdot 10923$ & 010101010101010101010101010101 \\
\hline 1665535 & 00000000000000001111111111111111 \\
\hline $16711935=255 \cdot 65537$ & 0000000011111111000000001111111 \\
\hline $252645135=3855 \cdot 65537$ & 00001111000011110000111100001111 \\
\hline $858993459=13107 \cdot 65537$ & 00110011001100110011001100110011 \\
\hline $1431655765=21845 \cdot 65537$ & 01010101010101010101010101010101 \\
\hline 17131071 & 0000000000000000011111111111111111 \\
\hline $393213=131071 \cdot 3$ & 0000000000000001011111111111111101 \\
\hline $5726623061=131071 \cdot 43691$ & 010101010101010101010101010101010 \\
\hline 18262143 & 000000000000000000111111111111111111 \\
\hline $1310715=262143 \cdot 5$ & 000000000000000100111111111111111011 \\
\hline $3407859=262143 \cdot 13$ & 000000000000001100111111111111110011 \\
\hline $9699291=262143.37$ & 000000000000100100111111111111011011 \\
\hline $17039295=262143.65$ & 000000000001000000111111111110111111 \\
\hline $28573587=262143 \cdot 109$ & 000000000001101100111111111110010011 \\
\hline $48496455=262143 \cdot 185$ & 000000000010111000111111111101000111 \\
\hline $126090783=262143 \cdot 481$ & 000000000111100000111111111000011111 \\
\hline $133956095=511 \cdot 262145$ & 000000000111111111000000000111111111 \\
\hline $142867935=262143 \cdot 545$ & 0000000010001000001111111110111011111 \\
\hline $241120971=4599 \cdot 52429^{*}$ & 000000001110010111110011011011001011 \\
\hline $371456631=262143 \cdot 1417$ & 000000010110001000111111101001110111 \\
\hline $401868285=1533 \cdot 262145$ & 000000010111111101000000010111111101 \\
\hline $630453915=262143 \cdot 2405$ & 000000100101100100111111011010011011 \\
\hline $1057222719=262143.4033$ & 0000001111110000001111111000000111111 \\
\hline $1205604855=4599 \cdot 262145$ & 000001000111110111000001000111110111 \\
\hline $1857283155=262143 \cdot 7085$ & 000001101110101100111110010001010011 \\
\hline $2545165805=9709 \cdot 262145$ & 000010010111101101000010010111101101 \\
\hline $3616814565=13797 \cdot 262145$ & 000011010111100101000011010111100101 \\
\hline $5286113595=262143 \cdot 20165$ & 000100111011000100111011000100111011 \\
\hline $7635497415=29127 \cdot 262145$ & 000111000111000111000111000111000111 \\
\hline $13743895347=262143 \cdot 52429$ & 001100110011001100110011001100110011 \\
\hline $22906492245=87381 \cdot 262145$ & 010101010101010101010101010101010101 \\
\hline 19524287 & 000000000000000000011111111111111111111 \\
\hline $1572861=524287 \cdot 3$ & 00000000000000000101111111111111111101 \\
\hline $91625968981=524287 \cdot 174763$ & 01010101010101010101010101010101010101 \\
\hline 201048575 & 0000000000000000000011111111111111111111 \\
\hline $17825775=1048575 \cdot 17$ & 0000000000000001000011111111111111101111 \\
\hline $1072694271=1023 \cdot 1048577$ & 00000000001111111111100000000001111111111 \\
\hline $5363471355=5115 \cdot 1048577$ & 00000001001111111101100000001001111111011 \\
\hline $26817356775=255$ & 111000001100011111 \\
\hline
\end{tabular}




\begin{tabular}{|c|c|}
\hline \multicolumn{2}{|c|}{ Делители $d$ числа $2^{n}-1$, принадлежащие $M(\lambda), n=2 \lambda$ (продолжение) } \\
\hline$\lambda \mid$ Варианты $d \in D(\lambda)$ & Двоичная запись $d$ \\
\hline 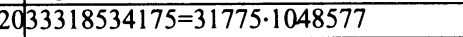 & 000001111100000111110000011 \\
\hline $43980465111=41943 \cdot 1048577$ & 000010100011110101110000101000 \\
\hline $64677154575=1048575 \cdot 61681$ & 0000111100001111000011110000111100001111 \\
\hline $99955602525=95325 \cdot 1048577$ & 0001011101000101110100010111010001011101 \\
\hline $219902325555=209715 \cdot 1048577$ & 0011001100110011001100110011001100110011 \\
\hline $366503875925=349525 \cdot 1048577$ & 0101010101010101010101010101010101010101 \\
\hline 212097151 & 000000000000000000000111111111111111111111 \\
\hline $6291453=2097151 \cdot 3$ & 00000000000000000001011111111111111111110 \\
\hline $18874359=2097151.9$ & 00000000000000000100011111111111111111011 \\
\hline $90177493=2097151 \cdot 43$ & 00000000000000010101011111111111111010101 \\
\hline $270532479=2097151 \cdot 129$ & 00000000000001000000011111 \\
\hline $437=2097151 \cdot 387$ & 00000000000011 \\
\hline $11364461269=2097151 \cdot 5419$ & 0000000010101001010101111111101 \\
\hline $34093383807=2097151 \cdot 16257$ & 000000011111110000000111111100000001111111 \\
\hline$\longdiv { 1 5 1 4 2 1 = 2 0 9 7 1 5 1 }$ & 00000101111101000001011111 \\
\hline $34567=$ & 0001110001110 \\
\hline $1466015503701=209715$ & 01010101010101 \\
\hline 224194 & 11 \\
\hline $15=4194303 \cdot 5$ & 00000000000000000001001111111 \\
\hline $8291=4194303 \cdot 397$ & 0000000000000110 \\
\hline $03 \cdot 1985$ & 00000000000 \\
\hline $8585742335=2047 \cdot 4194305$ & 000000000001111 \\
\hline $2239=4194303 \cdot 2113$ & 0000000000 \\
\hline $25757227005=6141 \cdot 4194305$ & 0000000001011111111101000000 \\
\hline $1195=4194303$ & 00000 \\
\hline $3607=60787 \cdot 8$ & 00000 \\
\hline $254959218035=60787 \cdot 4194305$ & 000000 \\
\hline $3518437208883=4194303 \cdot 838861$ & 00110 \\
\hline $5864062014805=139810$ & 010101010101010 \\
\hline 238388 & 0000 \\
\hline 2516 & $\overline{00000000}$ \\
\hline $9107643=17848$ & 0000 \\
\hline $23456248059221=8388607 \cdot 2796203$ & 01010101010101010 \\
\hline 2416777215 & 000000000000000000 \\
\hline$-10 / 1 / 213.911$ & 0000000000 \\
\hline $4311744255=16777215 \cdot 257$ & 00000000000000010000 \\
\hline $11291065695=16777215 \cdot 673$ & 000000000000001 \\
\hline $095 \cdot 167$ & 00000000000011 \\
\hline $92735=167772$ & 0000000001 \\
\hline $1095233372415=16777215 \cdot 65281$ & 0000000011 \\
\hline $1167945961455=69615$ & 0000000100 \\
\hline 172961 & 11111 \\
\hline 777217 & \\
\hline $16557351571215=986895 \cdot 16777217$ & 0000111100001 \\
\hline $21651921285435=1290555 \cdot 16777217$ & 0001001110 \\
\hline $31274997412295=1864135 \cdot 16777217$ & 00011100011100011100011100011100011 \\
\hline $56294995342131=33554$ & 1100110011 \\
\hline $92236885=5592405 \cdot 16777217$ & 0101 \\
\hline$2 5 \longdiv { 3 3 5 5 }$ & \\
\hline $293=33554431 \cdot 3$ & 000000000000 \\
\hline $369098741=33554431 \cdot 11$ & 0000000000000000 \\
\hline $1107296223=33554431 \cdot 33$ & 0000000000000000000100000111111111 \\
\hline $8422162181=33554431 \cdot 251$ & 0000000000000000011111010111111111111111100000101 \\
\hline $25266486543=33554431 \cdot 753$ & 0000000000000 \\
\hline $92643783991=33554431 \cdot 2761$ & 00000000000010 \\
\hline $135928999981=33554431 \cdot 4051$ & \\
\hline $51973=33554431 \cdot 8283$ & \\
\hline 2153 & 00000 \\
\hline
\end{tabular}




\begin{tabular}{|c|c|}
\hline \multicolumn{2}{|c|}{ Делители $d$ числа $2^{n}-1$, принадлежащие $M(\lambda), n=2 \lambda$ (продолжение) } \\
\hline$\lambda \mid$ Варианты $d \in D(\lambda)$ & Двоичная запись $d$ \\
\hline $25 \sqrt{1495218999791=33554431 \cdot 44561}$ & 0000000001010111000010000111111111010100011110111 \\
\hline $4485656999373=33554431 \cdot 133683$ & 00000001000001010001100101111111011111010111001101 \\
\hline $34118178995231=33554431 \cdot 1016801$ & 00000111110000011111000001111100000111110000011111 \\
\hline $102354536985693=33554431 \cdot 3050403$ & 00010111010001011101000101110100010111010001011101 \\
\hline $375299968947541=33554431 \cdot 11184811$ & 01010101010101010101010101010101010101010101010101 \\
\hline 2667108863 & 0000000000000000000000000011111111111111111111111111 \\
\hline $335544315=67108863 \cdot 5$ & 0000000000000000000000010011111111111111111111111011 \\
\hline $3556769739=67108863 \cdot 53$ & 0000000000000000000011010011111111111111111111100101 \\
\hline $10536091491=67108863 \cdot 157$ & 0000000000000000001001110011111111111111111101100011 \\
\hline $17783848695=67108863 \cdot 265$ & 00000000000000000100001000111111111111111111011 \\
\hline $52680457455=67108863 \cdot 785$ & 0000000000000000110001000011111111111111110011101111 \\
\hline $108246596019=67108863 \cdot 1613$ & 0000000000000001100100110011111111111111100110110011 \\
\hline $541232980095=67108863 \cdot 8065$ & 0000000000000111111000000011111111111110000001111111 \\
\hline $549688713215=8191 \cdot 67108865$ & 0000000000000111111111111100000000000001111111111111 \\
\hline $558412849023=67108863 \cdot 8321$ & 0000000000001000001000000011111111111101111101111111 \\
\hline $1649066139645=24573 \cdot 67108865$ & 0000000000010111111111110100000000000101111111111101 \\
\hline $2792064245115=67108863 \cdot 41605$ & 00000000001010001010000100 \\
\hline $5737069589007=67108863 \cdot 85489$ & 0000000001010011011111000011111111101011001000001111 \\
\hline $16994715574983=67108863 \cdot 253241$ & 0000000011110111010011100011111111000010001011 \\
\hline $28685347945035=67108863 \cdot 427445$ & 0000000110100001011011010011111110010111101001001011 \\
\hline $84973577874915=67108863 \cdot 1266205$ & 0000010011010100100001110011111011001010110111100011 \\
\hline $900719925474099=67108863 \cdot 13421773$ & 0011001100110011001100110011001100110011001100110011 \\
\hline $1501199875790165=22369621 \cdot 67108865$ & 0101010101010101010101010101010101010101010101010101 \\
\hline$2 7 \longdiv { 1 3 4 2 1 7 7 2 7 }$ & 0000000000000000000 \\
\hline $402653181=134217727 \cdot 3$ & 0000000000000000000000000101111111111111111111111111101 \\
\hline $1207959543=134217727 \cdot 9$ & 000000000000001000 \\
\hline $2550136813=134217727 \cdot 19$ & 000000000000000000000010010111111111111111111111101101 \\
\hline $3623878629=134217727 \cdot 27$ & 00000000000000000000001101011111111111111111111111100101 \\
\hline $7650410439=134217727 \cdot 57$ & 000000000000000000000111000111111111111111111111000111 \\
\hline $10871635887=134217727 \cdot 81$ & 000000000000000000001010000111111111111111111110101111 \\
\hline $22951231317=134217727 \cdot 171$ & 0000000000000000000101010101111111111111111111101010101 \\
\hline $68853693951=134217727 \cdot 513$ & 0000000000000000010000000001111111111111111110111111111 \\
\hline $206561081853=134217727 \cdot 1539$ & 0000000 \\
\hline $11705262189397=134217727 \cdot 87211$ & 000000000010101010010101010111111111101010101101010101 \\
\hline $35115786568191=134217727 \cdot 261633$ & 00000000011111111110000000001111111110000000001111111111 \\
\hline $105347359704573=134217727 \cdot 784899$ & 0000000101111111101000000010111111101000000010111111101 \\
\hline $222399981598543=134217727 \cdot 1657009$ & 000000110010100100010110000111111001101011011101001111 \\
\hline $316042079113719=134217727 \cdot 2354697$ & 0111000001000111110111000001000111110111 \\
\hline $667199944795629=134217727 \cdot 4971027$ & 000010010111101101000010010111101101000010010111101101 \\
\hline $948126237341157=134217727 \cdot 7064091$ & 000011010111100101000011010111100101000011010111100101 \\
\hline $2001599834386887=134217727 \cdot 14913081$ & 000111000111000111000111000111000111000111000111000111 \\
\hline $6004799503160661=134217727 \cdot 44739243$ & 010101010101010101010101010101010101010101010101010101 \\
\hline 28268435455 & 00000000000000000000000000001111111111111111111111111111 \\
\hline $4563402735=268435455 \cdot 17$ & 00000000000000000000000100001111111111111111111111101111 \\
\hline $4397778092031=16383 \cdot 268435457$ & 000000000000001111111111111110000000000000011111111111111 \\
\hline $21988890460155=81915 \cdot 268435457$ & 000000000001001111111111110110000000000010011111111111011 \\
\hline $127535564668899=475107 \cdot 268435457$ & 00000000011100111111111000110000000001110011111111100011 \\
\hline $496948924399503=1851279 \cdot 268435457$ & 00000001110000111111100011110000000111000011111110001111 \\
\hline $558586000294015=2080895 \cdot 268435457$ & 000000011111110000000111111110000000111111100000001111111 \\
\hline $637677823344495=2375535 \cdot 268435457$ & 00000010010000111111011011110000001001000011111101101111 \\
\hline $1675758000882045=6242685 \cdot 268435457$ & 00000101111101000001011111010000010111110100000101111101 \\
\hline $2484744621997515=9256395 \cdot 268435457$ & 0000100011010011110111001011000010001101001111011100101 \\
\hline $4238682002231055=268435455 \cdot 15790321$ & 00001111000011110000111100001111000011110000111100001111 \\
\hline $14411518807585587=53687091 \cdot 268435457$ & 00110011001100110011001100110011001100110011001100110011 \\
\hline $24019198012642645=89478485 \cdot 268435457$ & 01010101010101010101010101010101010101010101010101010101 \\
\hline 29536870911 & 000000000000000000000000000001111111111111111111111111111111 \\
\hline $1610612733=536870911 \cdot 3$ & 00000000000000000000000000010111111111111111111111111111101 \\
\hline $31675383749=536870911 \cdot 59$ & 000000000000000000000001110101111111111111111111111111000101 \\
\hline
\end{tabular}




\begin{tabular}{|c|c|}
\hline \multicolumn{2}{|c|}{ Делители $d$ числа $2^{n}-1$, принадлежащие $M(\lambda), n=2 \lambda$ (продолжение) } \\
\hline$\lambda$ Варианты $d \in D(\lambda)$ & Двоичная запись $d$ \\
\hline $2995026151247=536870911 \cdot 177$ & 00000000000000000000010110000111111111111111111111101001111 \\
\hline $1628420204246959=536870911 \cdot 3033169$ & 0000000101110010010000101000011111110100011011011110101111 \\
\hline $4885260612740877=536870911.9099507$ & 78000010001010110110001111001011111011101010010011100001101 \\
\hline 96076792050570581 & \\
\hline$=536870911 \cdot 178956971$ & 0101010101010101010101010101010101010101010101010101010101 \\
\hline 301073741823 & 000000000000000000000000000001111111111111111111111111111111 \\
\hline $5368709115=1073741823 \cdot 5$ & 000000000000000000000000001001111111111111111111111111111011 \\
\hline $13958643699=1073741823 \cdot 13$ & 00000000000000000000000001100111111111111111111111111110011 \\
\hline $26843545575=1073741823 \cdot 25$ & 000000000000000000000000110001111111111111111111111111100111 \\
\hline $44023414743=1073741823.41$ & 000000000000000000000001010001111111111111111111111111010111 \\
\hline $65498251203=1073741823 \cdot 61$ & 000000000000000000000001111001111111111111111111111111000011 \\
\hline $69793218495=1073741823 \cdot 65$ & 000000000000000000000001000000111111111111111111111110111111 \\
\hline $220117073715=1073741823 \cdot 205$ & 000000000000000000000011001100111111111111111111111100110011 \\
\hline $327491256015=1073741823 \cdot 305$ & 000000000000000000001001100001111111111111111111111011001111 \\
\hline $348966092475=1073741823 \cdot 325$ & 000000000000000000001010001001111111111111111111111010111011 \\
\hline $572304391659=1073741823 \cdot 533$ & 000000000000000000010000101001111111111111111111110111101011 \\
\hline $851477265639=1073741823.793$ & 000000000000000000001100011000111111111111111111110011100111 \\
\hline $1100585368575=1073741823 \cdot 1025$ & 00010000000000111111111111111111110111 \\
\hline $1418412948183=1073741823 \cdot 1321$ & $0000000000000000000101001010001 \mathrm{i} 11111111111111111101011$ \\
\hline $1637456280075=1073741823 \cdot 1525$ & 000000000000000000010111110100111111111111111111101000001011 \\
\hline $2685428299323=1073741823 \cdot 2501$ & 0000100111000100111111111111111 \\
\hline $58295=107$ & 0101001101000111111111111111 \\
\hline $4257386328195=1073741823 \cdot 3965$ & 000000000000000001111011111001111111111111111111000010000011 \\
\hline $7092064740915=1073741823 \cdot 6605$ & 000000000000000011001110011001111111111111111110011000110011 \\
\hline $13427141496615=1073741823 \cdot 12505$ & 000000000000000110000110110001111111111111111100111100100111 \\
\hline $14307609791475=1073741823 \cdot 13325$ & 0110100000011001111111 \\
\hline $18439368326379=10$ & 0001100010100111111111111 \\
\hline $21286931640975=10$ & 00000 \\
\hline $34910567891199=1073741823 \cdot 32513$ & 000000000000000111111100000000111111111111111000000011111111 \\
\hline 1073741825 & 00000000000000 \\
\hline $41823 \cdot 33025$ & 0000100000000 \\
\hline $741823 \cdot 54161$ & nola \\
\hline $741823 \cdot 62525$ & 000000000000001111010000111100111111111111110000101111000011 \\
\hline $823 \cdot 80581$ & 0000 \\
\hline $92196841631895=10$ & 00000 \\
\hline $105549895139325=98$ & 111111111110100000000000001011111111 \\
\hline $174552839455995=10$ & 1001111011000001001111111111110110000 \\
\hline $377515=1$ & 1000101011 \\
\hline $316649685417975=2$ & 0000 \\
\hline $387016282177525=3$ & 1111111110101000000000001010111 \\
\hline $9195815=1$ & 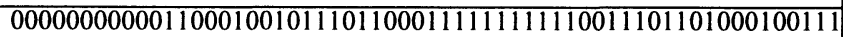 \\
\hline 46098 & 110011 \\
\hline $81539=1$ & 00011 \\
\hline $872764197279975=10$ & 00000 \\
\hline 1124801467909119 & \\
\hline$=1073741823 \cdot 10$ & 000000000011111111110000000000111111111100000000001111111111 \\
\hline$\overline{116104}$ & \\
\hline$=1$ & 0000000010 \\
\hline 887575 & \\
\hline $4,1823 \cdot 13$ & 00000000101001010100100101000111111111010110101011011010111 \\
\hline 2163079745979075 & \\
\hline$=1073741823 \cdot 2$ & 000000000111101011110100111100111111111000010100001011000011 \\
\hline 2687462714701275 & \\
\hline$=32537631.825$ & 000000001001100011000011101110101100011101001111010111011011 \\
\hline 299460 & \\
\hline$=13944699 \cdot 214748365^{*}$ & 000000001010101000111001001011110011010111011100000101111111 \\
\hline 3483146539597725 & \\
\hline$=3243933 \cdot 1073$ & 1110011101000000001100010111111110011101 \\
\hline
\end{tabular}




\begin{tabular}{|c|c|}
\hline \multicolumn{2}{|c|}{ Делители $d$ числа $2^{n}-1$, принадлежащие $M(\lambda), n=2 \lambda$ (продолжение) } \\
\hline$\lambda$ Варианты $d \in D(\lambda)$ & Двоичная запись $d$ \\
\hline \multicolumn{2}{|l|}{$3 0 \longdiv { 3 5 4 7 4 5 0 7 8 3 4 0 5 6 8 3 }$} \\
\hline$=1073741823 \cdot 3303821$ & 000000001100100110100110001100111111110011011001011001110011 \\
\hline \multicolumn{2}{|l|}{3780070506907695} \\
\hline$=1073741823 \cdot 3520465$ & 000000001101011011011111010000111111110010100100100000101111 \\
\hline \multicolumn{2}{|l|}{5624007339545595} \\
\hline$=1073741823.5237765$ & 000000010011111110110000000100111111101100000001001111111011 \\
\hline \multicolumn{2}{|l|}{11645671763705525} \\
\hline$=10845877 \cdot 1073741825$ & 000000101001010111111010110101000000101001010111111010110101 \\
\hline \multicolumn{2}{|c|}{14973006553335675} \\
\hline$=13944699 \cdot 1073741825$ & 000000110101001100011101111011000000110101001100011101111011 \\
\hline \multicolumn{2}{|l|}{17737253917028415} \\
\hline$=1073741823 \cdot 16519105$ & 000000111111000000111111000000111111000000111111000000111111 \\
\hline \multicolumn{2}{|c|}{18900352534538475} \\
\hline$=1073741823 \cdot 17602325$ & 000001000011001001011100010100111110111100110110100011101011 \\
\hline \multicolumn{2}{|l|}{28120036697727975} \\
\hline$=1073741823 \cdot 26188825$ & 000001100011111001110000011000111110011100000110001111100111 \\
\hline \multicolumn{2}{|c|}{34937015291116575} \\
\hline$=32537631 \cdot 1073741825$ & 000001111100000111110000011111000001111100000111110000011111 \\
\hline \multicolumn{2}{|l|}{46116860184273879} \\
\hline$=1073741823 \cdot 42949673$ & 000010100011110101110000101000111101011100001010001111010111 \\
\hline \multicolumn{2}{|l|}{88686269585142075} \\
\hline$=1073741823 \cdot 82595525$ & 000100111011000100111011000100111011000100111011000100111011 \\
\hline \multicolumn{2}{|l|}{104811045873349725} \\
\hline$=97612893 \cdot 1073741825$ & 000101110100010111010001011101000101110100010111010001011101 \\
\hline \multicolumn{2}{|l|}{128102389400760775} \\
\hline \multicolumn{2}{|c|}{$=119304647 \cdot 1073741825000111000111000111000111000111000111000111000111000111000111$} \\
\hline \multicolumn{2}{|l|}{230584300921369395} \\
\hline \multicolumn{2}{|l|}{$=1073741823 \cdot 2147$} \\
\hline \multicolumn{2}{|l|}{384307168202282325} \\
\hline$=357913941 \cdot 1073$ & 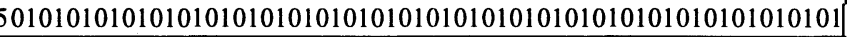 \\
\hline
\end{tabular}

\section{5. Заключение}

Помимо значений $n=2 \lambda$, удовлетворяющих условию предложения 6 , существуют также и другие значения $n$, для которых все числа из $M(\lambda)$ делятся на $2^{\lambda}-1$, и следовательно, все гипер-бент-функции от $n$ переменных описываются теоремой 3 . Так, из приведенной выше табл. 1 видно, что, помимо чисел $n=2 \lambda \in\{6,10,14,26,34,38,58\}$, для которых $2^{\lambda}-1-$ простое число, это условие выполняется также для чисел $n \in\{18,50,54\}$.

Компьютерные вычисления показали, что существует точно 18 гипер-бент-функций от $n=4$ переменных, сохраняющих 0 и не удовлетворяющих условию теоремы 3 .

Более того, компьютерные вычисления показали также, что, хотя множество $M(4)$ и содержит числа, не делящиеся на $2^{\lambda}-1=15$, тем не менее, все гипер-бент-функции от 8 переменных суть в точности гипер-бент-функции, описанные теоремой 3.

Вопрос, существуют ли гипер-бент-функщии от $n>4$ переменных, не удовлетворяющие условию теоремы 3 , остается открытым. 
Таблица 2. ГБ-функции от $n=4$ переменных, сохраняющие 0

Согласно табл. 1, справедливо равенство $D(2)=\{3,5\}$. Несложные вычисления с помощью следствия 1 показывают, что $M(2)=D(2)$. Пусть $\theta \in Q=G F(4)$ - примитивный элемент, являющийся корнем многочлена $x^{4}+x+1$. Тогда согласно теореме 2 любая ГБ-функция $F: Q \rightarrow P, F(0)=0$, однозначно представляется в виде

$$
F(x)=\operatorname{tr}_{P}^{Q}\left(c_{3} \xi_{3} x^{3}+c_{5} \xi_{5} x^{5}\right),
$$

где $\xi_{3}=1, c_{3} \in Q_{3}=Q, \xi_{5}=\theta, c_{5} \in Q_{5}=G F(2)$. Ниже приводятся пары значений $c_{3}, c_{5}$ и соответствующие наборы значений $\left(F(0), F(1), F(\theta), \ldots, F\left(\theta^{14}\right)\right.$, при которых функция (4.5) есть ГБ-функция. При этом значение $c_{r}$ задается числом $a_{0}+2 a_{1}+4 a_{2}+8 a_{3}$, которое соответствует представлению

$$
c_{r}=a_{0}+a_{1} \theta+a_{2} \theta^{2}+a_{3} \theta^{3}, \quad a_{0}, a_{1}, a_{2}, a_{3} \in G F(2) .
$$

Ситуации, когда $c_{5}=0$, соответствуют ГБ-функциям, которые описаны теоремой 3 .

\begin{tabular}{|c|c|c||c|c|c|}
\hline$c_{3}$ & $c_{5}$ & Значения ГБ & $c_{3}$ & $c_{5}$ & Значения ГБФ \\
\hline 0 & 1 & 00011011011011011 & 12 & 6 & 00001101000001011 \\
0 & 6 & 00110110110110110 & 12 & 7 & 00010110011010000 \\
0 & 7 & 00101101101101101 & 11 & 0 & 00101001010010100 \\
1 & 1 & 00000101100110100 & 5 & 0 & 00011000110001100 \\
1 & 6 & 00101000001011001 & 10 & 1 & 00101100110100000 \\
1 & 7 & 00110011010000010 & 10 & 6 & 00000001011001101 \\
2 & 0 & 00001010010100101 & 10 & 7 & 00011010000010110 \\
4 & 0 & 00000110001100011 & 7 & 0 & 00010010100101001 \\
8 & 1 & 00100110100000101 & 14 & 0 & 00110001100011000 \\
8 & 6 & 00001011001101000 & 15 & 1 & 00110100000101100 \\
8 & 7 & 00010000010110011 & 15 & 6 & 00011001101000001 \\
3 & 0 & 00010100101001010 & 15 & 7 & 00000010110011010 \\
6 & 0 & 00001100011000110 & 13 & 0 & 00100101001010010 \\
12 & 1 & 00100000101100110 & 9 & 0 & 00100011000110001 \\
\hline
\end{tabular}

\section{Список литературы}

1. Golomb S. W., On the classification of Boolean functions. Trans. JRE CT-6, 1959.

2. Zierler N., Mills W. H., Products of linear recurring sequences. J. Algebra (1973) 27, №1, 147-157.

3. Rothaus O. S., On "bent” functions. J. Comb. Theory, Ser. A (1976) 20, №3, 300-305.

4. Dilon J. F., A survey of bent functions. NSA Technical J. (1972) 191-215.

5. Gong G., Golomb S. W., Transform domain analysis of DES. Techn. Rep., Univ. Waterloo, Canada, 1998.

6. Глухов М. М., Елизаров В. П., Нечаев А. А., Алгебра, ч. 2. Гелиос, Москва, 2003.

7. Lam T. Y., A first course in noncommutative rings. Springer, Berlin, 1991.

8. Лидл Р., Нидеррайтер Г., Конечные поля, т. 1, 2. Мир, Москва, 1988.

9. Мак-Вильямс Ф. Дж., Слоэн Н. Дж., Теория кодов, исправляющих ошибки. Связь, Москва, 1979.

10. Логачев О. А., Сальников А. А., Ященко В. В., О свойствах сумм Вейля на конечных полях и конечных абелевых группах. Дискретная математика (1999) 11, №2, 66-85.

11. Постников М. М., Введение в теорию алгебраических чисел. Наука, Москва, 1982.

12. Youssef A. M., Gong G., Hyper-bent functions. Lect. Notes Comput. Sci. (2001) 2045, 406-419.

Статья поступила 25.01.2006. 